05

\title{
Анизотропия гигантских нелинейных магнитоэлектрических эффектов в гексаферритах со структурой магнитоплюмбита
}

\author{
(C) М.А. Попов, И.В. Зависляк
}

Киевский национальный университет им. Тараса Шевченко, 01601 Киев, Украина

e-mail: maxim_popov@univ.kiev.ua

(Поступило в Редакцию 24 ноября 2017 г.)

\begin{abstract}
Представлены результаты исследования анизотропии нелинейного магнитоэлектрического токового эффекта в гексаферритах $M$-типа с коллинеарным магнитным упорядочением при комнатной температуре. Обнаружено, что при протекании постоянного электрического тока в плоскости, перпендикулярной к гексагональной оси образца гексаферрита стронция, уменьшаются намагниченность насыщения и константа анизотропии материала. Изменение магнитных параметров, измеренное методами радиоспектроскопии, оказалось пропорционально плотности подводимой электрической мощности, причем найденные коэффициенты пропорциональности были в разы больше по сравнению со случаем протекания тока вдоль оси. Предложена феноменологическая теория, качественно объясняющая наблюдаемые эффекты.
\end{abstract}

DOI: $10.21883 /$ JTF.2018.08.46310.2578

\section{Введение}

Возможность управления магнитными параметрами магнитоупорядоченных материалов при помощи электрических сигналов и, наоборот, их электрическими свойствами при помощи магнитных полей давно привлекает внимание исследователей, поскольку представляет значительный фундаментальный и прикладной интерес.

Среди обнаруженных к данному времени эффектов электрического управления магнитными свойствами можно выделить две группы. В первом случае воздействие осуществляется за счет переноса спинового момента при протекании через образец спинполяризованного тока электронов [1-3]. При этом существенным ограничением данного подхода является необходимость использования высоких плотностей тока, достигающих $\approx 10^{7} \mathrm{~A} / \mathrm{cm}^{2}$. Вторая группа эффектов, называемых магнитоэлектрическими (МЭ), предусматривает непосредственное влияние электрического поля на намагниченность [4-6]. Они наблюдаются в мультиферроиках - однофазных или композитных материалах, в которых сосуществуют магнитное и электрическое упорядочения. Так как в однофазных мультиферроиках при комнатной температуре МЭ эффект обычно невелик, основное внимание уделяется многокомпонентным структурам ферромагнетик-пьезоэлектрик, в которых взаимодействие между фазами осуществляется через упругую подсистему [4]. В настоящей работе представлены результаты исследования нелинейных МЭ эффектов в гексаферрите стронция, который принадлежит к классу ферримагнетиков с коллинеарным упорядочением магнитных моментов подрешеток, а также является полупроводником $n$-типа [7], в котором в принципе возможны как чисто полевые, так и токовые эффекты. Линейный МЭ эффект в данном случае запрещен, так как в точечной группе кристалла присутствует центр инверсии, однако МЭ эффекты четных по электрическому полю порядков симметрией допускаются. Отметим, впрочем, что при наличии в гексаферрите геликоидальной магнитной структуры это ограничение снимается, и линейный МЭ эффект наблюдается экспериментально [8]. Применение гексаферритов в составе композитных материалов показало принципиальную возможность электрического управления резонансной частотой таких структур в миллиметровом диапазоне $[9,10]$, но полученные величины перестройки оказались слишком малыми для практического применения. Недавно в гексаферрите стронция были экспериментально обнаружены нелинейные МЭ токовые эффекты, которые по величине на порядок больше наблюдаемых в гетероструктурах [11]. Их можно назвать гигантскими по аналогии с гигантским магнитосопротивлением. Как будет показано ниже, при протекании тока в базисной кристаллографической плоскости образца вследствие анизотропии его свойств влияние билинейных МЭ эффектов на магнитные параметры материала в разы больше по сравнению с ситуацией, когда ток проходит вдоль гексагональной оси. При этом плотность управляющего тока остается на много порядков меньше характерных для эффектов переноса спинового момента величин.

\section{Феноменологическая модель}

Согласно [12], полная энергия магнитоэлектрического материала состоит из магнитного, электрического и магнитоэлектрического вкладов: $W=$ $=W_{E}\left(E, P_{S}\right)+W_{M}\left(H, M_{S}\right)+W_{M E}(E, H)$, где символами $P_{S}$ и $M_{S}$ обозначены спонтанные поляризация и намагниченность. Благодаря последнему слагаемому как поляризация $P_{i}=-\partial W / \partial E_{i}$, так и намагниченность $M_{i}=-\partial W / \partial H_{i}$ становятся функциями электрического и магнитного поля одновременно. Однако такая форма 
записи энергии не всегда адекватна для магнитоупорядоченных анизотропных материалов. В настоящей работе в качестве базового будет использовано выражение для объемной плотности магнитной свободной энергии анизотропного ферромагнетика, однородно намагниченного внешним магнитным полем $H_{0}$, вида $[13,14]$

$$
W_{M}=-\frac{1}{2} M_{i} H_{i}^{(m)}-M_{i} H_{0 i}+K_{i j} \alpha_{i} \alpha_{j}
$$

Энергия включает в себя диполь-дипольную, зеемановскую и спин-орбитальную $\left(\alpha_{i}=M_{i} /|M|, \quad K_{i j}-\right.$ константы анизотропии) составляющие. Размагничивающее поле можно представить как $H_{i}^{(m)}(r)=$ $=\int_{V} M_{k}\left(r^{\prime}\right) \frac{\partial^{2}}{\partial r_{i}^{\prime} \partial r_{k}^{\prime}}\left(1 /\left|r-r^{\prime}\right|\right) d r^{\prime}$ [13]. Для однородно намагниченных тел эллипсоидальной формы это выражение упрощается и принимает вид $H_{i}^{(m)}(r)=N_{i j} M_{j}(r)$, где $N_{i j}$ - тензор размагничивающих коэффициентов.

Рассмотрим далее образец в форме тонкой пластинки в ненасыщенном состоянии с полосовыми доменами равного объема и противоположно направленными векторами намагниченности при отсутствии внешнего магнитного поля. Если намагниченности доменов направлены перпендикулярно поверхности образца, то [14]

$$
W_{M}=\frac{\pi}{2}\left(M_{i}^{(1)} n_{i}-M_{i}^{(2)} n_{i}\right)^{2}+\frac{1}{2} K_{i j}\left(\alpha_{i}^{(1)} \alpha_{j}^{(1)}+\alpha_{i}^{(2)} \alpha_{j}^{(2)}\right) .
$$

Здесь $n$ - вектор нормали к доменной границе, верхний индекс обозначает разные типы доменов. Для феноменологического описания влияния электрического поля на магнитные параметры добавим к свободной энергии квадратичное по $E$ „магнитобиэлектрическое“ $[12,15]$ слагаемое в виде

$$
-W_{M B E}=\gamma_{i j k} E_{j} E_{k} B_{i}+\delta_{i j k l} E_{k} E_{l} \alpha_{i} \alpha_{j},
$$

где $B_{i}=H_{i}+4 \pi M_{i}$. Свойства входящих в (3) тензоров определяются кристаллографической симметрией ферромагнетика [15]. Здесь учтено, что кристаллическая структура гексаферритов $M$-типа содержит центр инверсии [16], а значит, линейные по электрическому полю слагаемые отсутствуют.

Определим теперь характерный вид введенных выше тензоров для гексаферритов $M$-типа с коллинеарной намагниченностью подрешеток (магнитная точечная группа 6/mm $\left.m^{\prime}\right)$. Используя принцип Неймана [16], можно показать, что тензор $\gamma_{i j k}$ инвариантен относительно всех симметрийных преобразований данной точечной группы и содержит лишь 7 ненулевых коэффициентов (из них 3 независимых):

$$
\gamma_{i l}=\left(\begin{array}{cccccc}
0 & 0 & 0 & 0 & \gamma_{15} & 0 \\
0 & 0 & 0 & \gamma_{15} & 0 & 0 \\
\gamma_{31} & \gamma_{31} & \gamma_{33} & 0 & 0 & 0
\end{array}\right)
$$

Здесь для второй пары индексов использована сокращенная запись $1=11 ; 2=22 ; 3=33 ; 4=23=32$;
$5=31=13 ; 6=12=21$, а ось 3 является осью симметрии 6-го порядка. Заметим, что по виду он совпадает с пьезомагнитным тензором данной точечной группы [16]. Это следствие того, что произведение $E_{j} E_{k}$ преобразуется при операциях симметрии так же, как и тензор упругих напряжений [12]. Тем же способом можно получить и общее выражение для второго введенного тензора, аналогичного теперь уже тензору магнитострикции [16]

$$
\delta_{m, n}=\left(\begin{array}{cccccc}
\delta_{11} & \delta_{21} & \delta_{13} & 0 & 0 & 0 \\
\delta_{21} & \delta_{11} & \delta_{13} & 0 & 0 & 0 \\
\delta_{31} & \delta_{31} & \delta_{33} & 0 & 0 & 0 \\
0 & 0 & 0 & \delta_{44} & 0 & 0 \\
0 & 0 & 0 & 0 & \delta_{44} & 0 \\
0 & 0 & 0 & 0 & 0 & \frac{\delta_{11}-\delta_{21}}{2}
\end{array}\right) .
$$

В нем имеется 21 ненулевой коэффициент (6 независимых). Здесь обозначения Фойгта использованы для обоих нижних индексов.

Рассмотрим теперь ситуацию, в которой внешнее магнитное поле и ось $z$ совпадают по направлению с кристаллографической осью симметрии. Тогда, используя (3) и (4), находим индуцированное электрическим полем изменение проекции намагниченности на направление магнитного поля:

$$
\Delta M_{z}=-\frac{\partial W_{M B E}}{\partial H_{i}}=\gamma_{311} E_{\perp}^{2}+\gamma_{333} E_{\|}^{2},
$$

где символами $\|$ и $\perp$ обозначены параллельные и ортогональные к оси симметрии компоненты соответствующих величин.

Проведя замену $E_{i}=\rho_{i j} J_{j}$, где $\rho_{i j}-$ тензор удельных сопротивлений, $\mathbf{J}$ - вектор плотности тока, и полагая, что тензор сопротивлений имеет анизотропный диагональный вид [17], изменение проекции намагниченности можно выразить через экспериментально измеряемую величину - подводимую электрическую мощность $P$ :

$$
\left(\Delta M_{z}\right)_{\perp}=\gamma_{311} \rho_{\perp} \frac{P}{V},\left(\Delta M_{z}\right)_{\|}=\gamma_{333} \rho_{\|} \frac{P}{V} .
$$

Здесь $P=U I=\rho J^{2} V$, а $V-$ объем образца, через который протекает ток.

Таким образом, изменение величины проекции намагниченности на ось $z$ для конкретного образца будет пропорционально приложенной мощности независимо от того, пропускается электрический ток вдоль оси симметрии гексаферрита или же перпендикулярно к ней.

Найдем теперь индуцированное электрическим полем изменение энергии анизотропии. Когда вектор $\mathbf{E}$ параллелен гексагональной оси, квадратичное магнитоэлектрическое слагаемое в $(2)$ имеет вид $\left(\delta_{3333}-\delta_{3311}\right) E_{\|}^{2} \alpha_{3}^{2}$. Если же ток протекает в плоскости, симметрия системы понижается. Для этого случая, оставляя только значимые члены, приходим к приближенному выражению для МЭ вклада: $\Delta K_{i j} \approx\left(\delta_{1133}-1 / 2\left(\delta_{1111}+\delta_{2211}\right)\right) E_{\perp}^{2} \alpha_{3}^{2}$, которое, впрочем, при $\delta_{1111}=\delta_{2211}$ становится точным. Учитывая, что для гексаферритов с анизотропией типа „легкая ось““ невозмущенное спин-орбитальное 
слагаемое в полной энергии имеет вид $W_{a}=-K_{u} \alpha_{3}^{2}$, $K_{u} \equiv K_{33}>0$ [18], приходим к окончательным выражениям для магнитоэлектрических поправок к константе анизотропии:

$$
\begin{gathered}
\left(\Delta K_{u}\right)_{\perp}=\left(\delta_{1133}-\frac{\delta_{1111}+\delta_{2211}}{2}\right) \rho_{\perp} \frac{P}{V}, \\
\left(\Delta K_{u}\right)_{\|}=\left(\delta_{3333}-\delta_{3311}\right) \rho_{\|} \frac{P}{V} .
\end{gathered}
$$

В заключение рассмотрим изменение такого магнитного параметра как эффективное поле одноосной анизотропии, определяемого выражением $H_{a}=2 K_{u} / M_{z}$ [18]. Поскольку $\Delta H_{a} / H_{a} \approx\left(\Delta K_{u} / K_{u}-\Delta M_{z} / M_{z}\right)$, то изменение поля анизотропии $\Delta H_{a}$ также будет линейно пропорционально подведенной электрической мощности, но при этом его знак может быть любым, в зависимости от соотношения между относительными изменениями величин $M_{z}$ и $K_{u}$.

\section{Экспериментальные результаты}

В ходе проведенных экспериментов исследовались монокристаллические образцы гексаферрита стронция $\mathrm{SrFe}_{12} \mathrm{O}_{19}$ в форме параллелепипедов толщиной $d=160 \mu \mathrm{m}$, вырезанных так, чтобы гексагональная ось была направлена перпендикулярно к развитой плоскости. На одну из поверхностей образцов методом магнетронного напыления через маску наносились две полоски из платины толщиной $0.4 \mu \mathrm{m}$ с подслоем из титана, служившие контактными площадками, при помощи которых образец припаивался к фольгированному стеклотекстолиту с поперечной щелью в месте расположения гексаферрита (рис. 1). Таким образом, при приложении

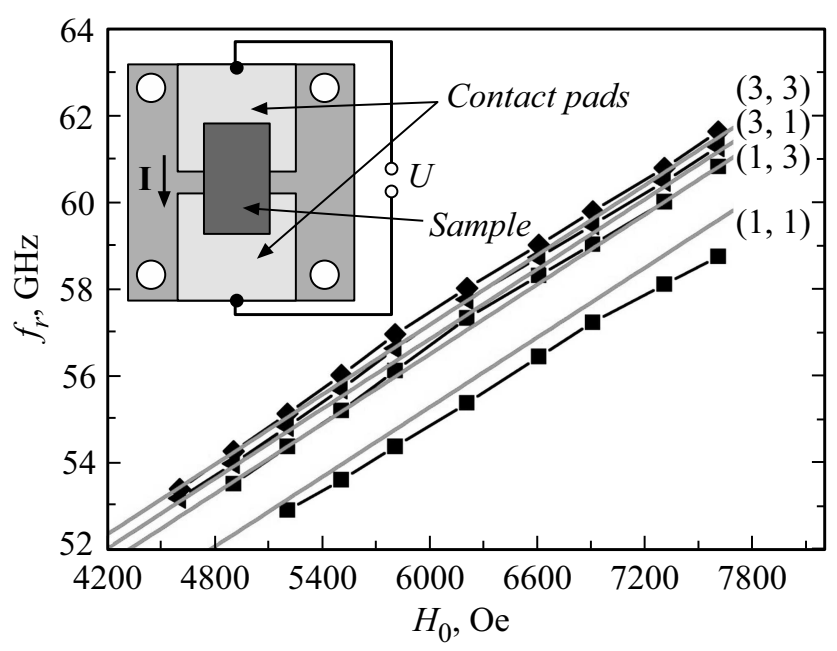

Рис. 1. Зависимость частот МСК в насыщенном образце гексаферрита стронция с размерами $1.4 \times 1.1 \times 0.16 \mathrm{~mm}$ от магнитного поля (точки - эксперимент, линии - расчет согласно (9)). На вставке изображена измерительная ячейка и показано направление протекания электрического тока через образец. к измерительной ячейке электрического напряжения через образец протекал электрический ток в плоскости, перпендикулярной к гексагональной оси, изучение влияния которого на магнитные свойства образца и было предметом исследований. Все эксперименты проводились при комнатной температуре. Изменение магнитных параметров образца регистрировалось радиоспектроскопическим методом по сдвигу частот магнитостатических колебаний (МСК). В ходе измерений описанная выше ячейка присоединялась к открытому концу прямоугольного металлического волновода миллиметрового диапазона через изолирующую диэлектрическую прокладку, после чего записывались амплитудно-частотные характеристики (АЧХ) коэффициента отражения $\left|S_{11}(f)\right|$ при разных значениях протекающего через образец тока. Сам волновод размещался между полюсными наконечниками электромагнита так, чтобы внешнее постоянное магнитное поле было направлено вдоль легкой оси гексаферрита, т.е. перпендикулярно плоскости образца. Измерения проводились при помощи скалярных измерителей КСВН и ослабления Р2-68 (диапазон частот $37.5-53.6 \mathrm{GHz})$ и $\mathrm{P} 2-69(53.6-78.3 \mathrm{GHz})$. Сначала исследовались зависимости частот наблюдаемых магнитостатических мод от приложенного магнитного поля при нулевом токе через образец в диапазоне от поля перехода в ненасыщенное (многодоменное) состояние до максимального поля, создаваемого электромагнитом. Эти данные (рис. 1) позволили определить значение гиромагнитного отношения $\gamma$ для гексаферрита по углу наклона частотно-полевых зависимостей, а также оценить намагниченность насыщения. Полученные значения $\gamma=2.68 \pm 0.03 \mathrm{MHz} / \mathrm{Oе} \mathrm{и} 4 \pi M_{0}=4590 \pm 50 \mathrm{G}$ находятся в хорошем соответствии с известными из литературы данными $[19,20]$.

Далее было исследовано влияние постоянного тока, протекающего в плоскости образца, на частоту магнитостатических колебаний. Для этого на образец подавался короткий (длительностью порядка $1 \mathrm{~s}$ ) импульс тока определенной амплитуды и записывалась АЧХ отраженного сигнала. Малая длительность импульса позволяла избежать существенного повышения температуры образца вследствие омического нагрева (проведенные отдельно измерения показали, что типичные скорости изменения частот МСК, из-за нагрева образца постоянным током, составляли не более $5 \mathrm{MHz} / \mathrm{s}$, таким образом, термический вклад в наблюдаемый импульсный эффект не превышал 3\%). Примеры полученных частотных зависимостей модуля коэффициента отражения при разных значениях постоянного тока приведены на рис. 2, $a$ и 3,a. Из них видно, что протекание тока приводит к сдвигу резонансных частот МСК, причем величина сдвига возрастает с увеличением тока. Причиной этого являются индуцированные протекающим током изменения магнитных параметров материала, как это описано в теоретической части. Максимальное значение пропускаемого тока было ограничено во избежание необратимого лавинного пробоя. Измерения проводи- 
лись в двух магнитных состояниях образца: ненасыщенном (при нулевом внешнем магнитном поле), характеризующемся наличием в образце доменных структур, и насыщенном, при котором в образце имеет место квазиоднородная намагниченность. Зависимости сдвига частот соответствующих мод от плотности подводимой к образцу электрической мощности в разных магнитных состояниях показаны на рис. $2, b$ и $3, b$. Как видно, в данном диапазоне значений мощности они являются линейными, при этом их крутизна составляет примерно $110 \mathrm{MHz} \cdot \mathrm{mm}^{3} / \mathrm{W}$ для $H_{0}=0$ и $390-440 \mathrm{MHz} \cdot \mathrm{mm}^{3} / \mathrm{W}$ для квазиоднородно намагниченного состояния.

Для интерпретации полученных результатов использовалась модель ферромагнитного резонанса (ФМР) в многодоменном состоянии [14,18]. Резонансный спектр при этом оказывается состоящим из двух мод: высокочастотной и низкочастотной. Низкочастотная мода (с синфазными колебаниями нормальных к доменной границе компонент намагниченности в соседних доменах) при
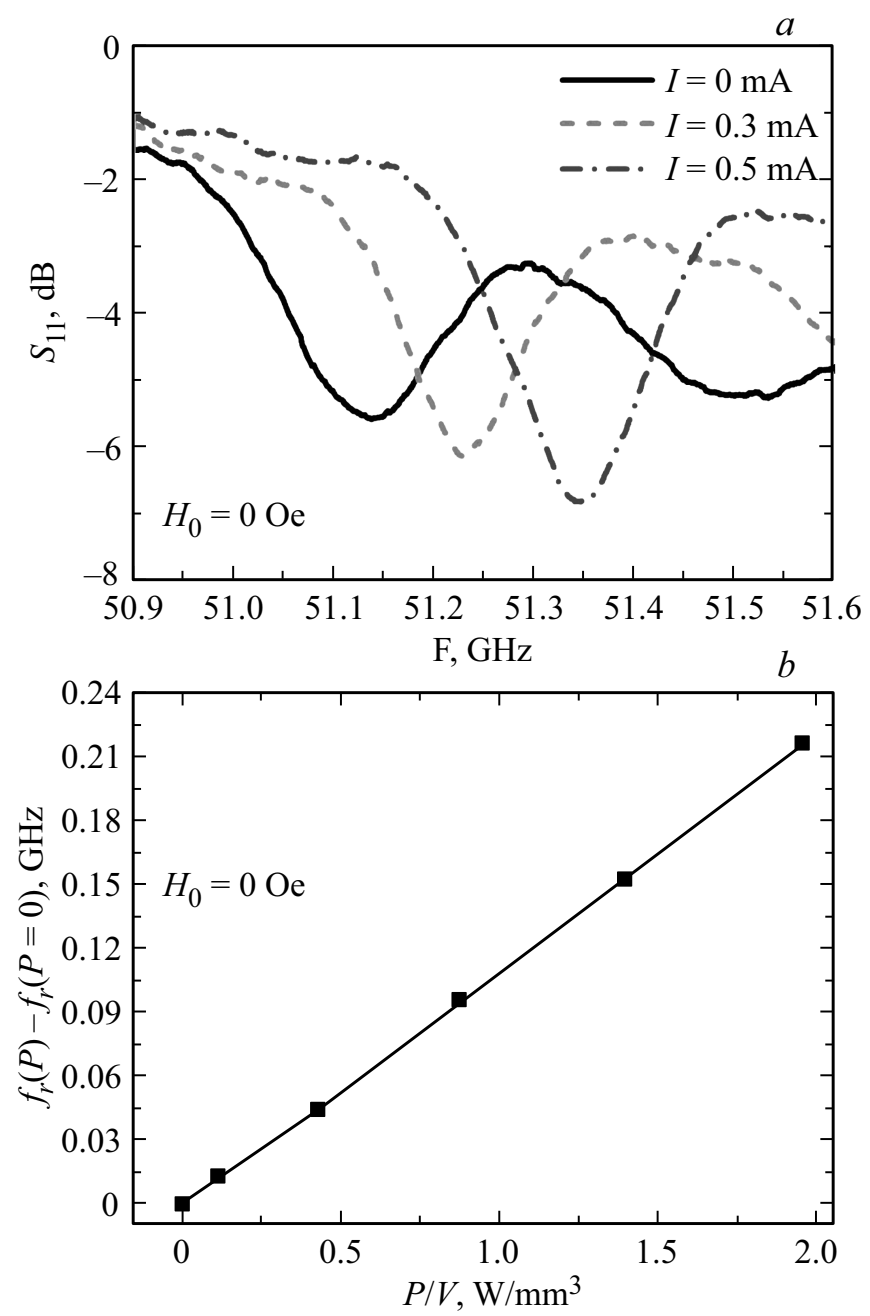

Pис. 2. Перестройка частоты низкочастотного доменного резонанса в гексаферрите стронция в многодоменном состоянии электрическим током; $a$ - частотные зависимости коэффициента отражения; $b-$ сдвиг резонансной частоты от плотности подводимой к образцу электрической мощности.
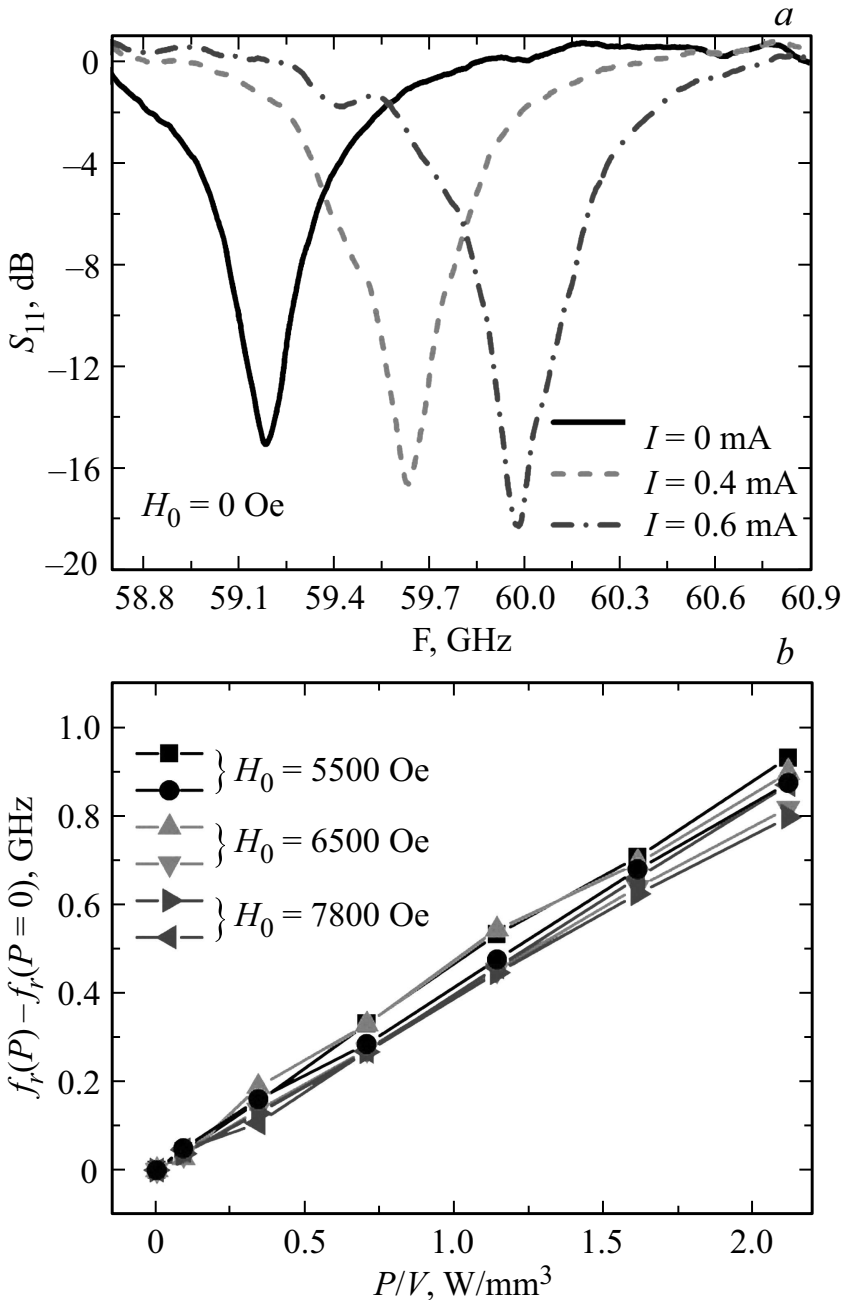

Рис. 3. Перестройка частот МСК в образце гексаферрита стронция в насыщенном состоянии электрическим током; $a-$ типичные частотные зависимости коэффициента отражения; $b$ - сдвиг резонансных частот разных МСК для нескольких значений постоянного магнитного поля от плотности подводимой электрической мощности.

$H_{0}=0$ имеет частоту [14]

$$
f_{r}\left(H_{0}=0\right)=\gamma H_{a},
$$

где $H_{a}$ - поле одноосной анизотропии, введенное выше. Именно эта мода удобна для измерений при нулевом внешнем поле, так как позволяет непосредственно определить индуцированное протекающим током изменение $H_{a}$. С другой стороны, в насыщенном состоянии частоты магнитостатических колебаний нормально намагниченного односторонне металлизированного слоя одноосного ферромагнетика определяются из соотношений [18]

$$
\begin{gathered}
|k| d=\frac{1}{\sqrt{-\mu}} \operatorname{arctg}\left(\frac{1}{\sqrt{-\mu}}\right), \\
\mu=\frac{f^{2}-f_{H}\left(f_{H}+f_{M}\right)}{f^{2}-f_{H}^{2}} .
\end{gathered}
$$




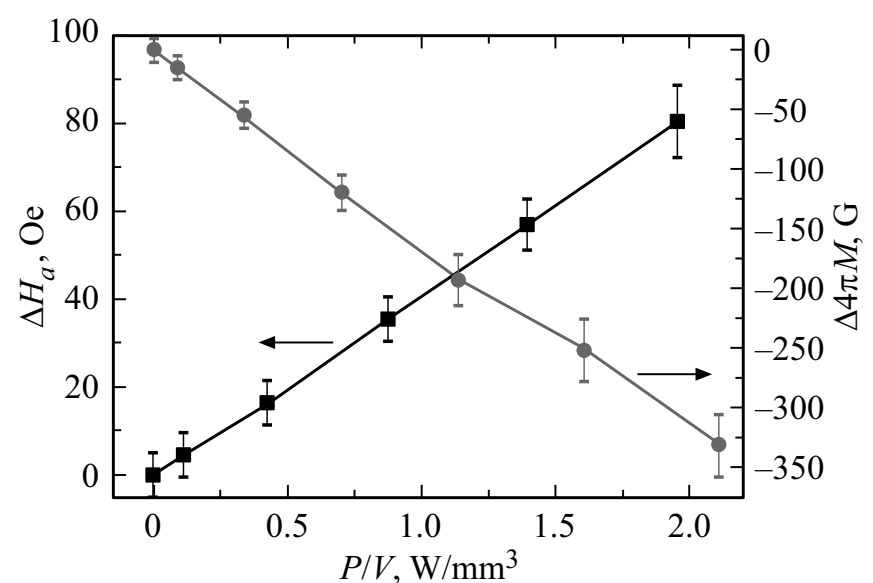

Рис. 4. Изменения магнитных параметров (намагниченность и эффективное поле одноосной анизотропии) гексаферрита стронция при пропускании электрического тока в плоскости образца как функция плотности подводимой электрической мощности.

Здесь $k$ - поперечное волновое число, определяемое размерами резонатора, $f_{H}=\gamma\left(H_{0}+H_{a}-N_{z z} 4 \pi M_{z}\right)$, $f_{M}=\gamma 4 \pi M_{z}, N_{z z}$ - эквивалентный размагничивающий фактор образца [21]. Для резонатора прямоугольной формы в приближении магнитной стенки можно положить $k^{\{n m\}}=\pi \sqrt{(n / a)^{2}+(m / b)^{2}}, n, m=1,2,3$ [18]. $\mathrm{B}$ дальнейшем индексы $(n, m)$ будут использоваться для обозначения отдельных мод МСК. Рассмотрим только основную ветвь решения трансцендентного дисперсионного уравнения (9), соответствующую максимальным значениям $\mu$. В этом случае каждой моде МСК с фиксированным $k=k^{\{n m\}}$ соответствует одно конкретное значение $\mu=\mu^{\{n m\}}$, которое определяется лишь геометрическими размерами резонатора. Тогда, считая поле $H_{0}$ постоянным и приравнивая к нулю полный дифференциал от $\mu^{\{n m\}}$ при условии, что $\left(f^{\{n m\}}-f_{H}\right) \ll f_{M}, f_{H}, f^{\{n m\}}$, получаем выражение для сдвига частоты МСК, вызванного изменениями магнитных параметров материала:

$$
\frac{\Delta f^{\{n m\}}}{\gamma} \approx \Delta H_{a}-N_{z z} \Delta\left(4 \pi M_{z}\right) .
$$

Из выражения (10) следует, что в рамках использованных приближений сдвиг частоты, во-первых, не зависит от номера моды и, во-вторых, не зависит от приложенного магнитного поля. На рис. $3, b$ показаны экспериментально измеренные сдвиги частот для пары нижайших мод (для которых указанное выше условие хорошо выполняется) при трех разных значениях поля $H_{0}$. Видно, что $\Delta f^{\{n m\}}$ в первом приближении действительно почти не зависит ни от индексов моды, ни от магнитного поля. Тогда, используя определенные ранее значения $\Delta H_{a}$ и формулу (10), из этих данных можно рассчитать вызванное током изменение намагниченности. Полученные значения $\Delta H_{a}$ и $\Delta\left(4 \pi M_{z}\right)$ приведены на рис. 4 (при этом показанные погрешности учитывают как инструментальные ошибки, так и нагрев образца). Используя их, находим, что намагниченность $M_{z}$ и константа анизотропии $K_{u}$ линейно зависят от плотности подводимой электрической мощности с коэффициентами пропорциональности $-(11.5 \pm 1.2) \mathrm{G} \cdot \mathrm{mm}^{3} / \mathrm{W}$ и $-(1160 \pm 120) \mathrm{erg} / \mathrm{W}$. $\mathrm{B}$ то же время для случая тока, протекающего вдоль гексагональной оси кристалла [11], соответствующие величины равны примерно $-2 \mathrm{G} \cdot \mathrm{mm}^{3} / \mathrm{W}$ и $-170 \mathrm{erg} / \mathrm{W}$, т.е. в 6-7 раз меньше. Заметим, что это соотношение („анизотропия МЭ эффекта“) оказывается одного порядка с анизотропией электрической проводимости в гексаферритах $M$-типа [17].

\section{Заключение}

Экспериментально и теоретически показана возможность индуцированных электрическим током нелинейных магнитоэлектрических эффектов в магнитоупорядоченном кристалле. Методами СВЧ радиоспектроскопии установлено, что протекание тока в монокристаллическом гексаферрите $M$-типа в плоскости, перпендикулярной к оси симметрии, вызывает уменьшение его статической намагниченности и константы одноосной анизотропии. При этом коэффициенты, характеризующие данные эффекты, оказались существенно (в 6-7 раз) больше, чем в исследованном ранее случае протекания тока вдоль гексагональной оси, что свидетельствует об их ярко выраженной анизотропии. Возможно, причиной данных эффектов являются частично делокализованные электроны проводимости, возникающие из-за наличия в кристалле четырехвалентных примесей. Под воздействием электрического поля эти электроны срываются со своих мест и участвуют в „прыжковой“ проводимости, передвигаясь в основном по бездефектным узлам с ионами $\mathrm{Fe}^{3+}$, временно переводя их в состояние $\mathrm{Fe}^{2+}$ с меньшим спиновым магнитным моментом и энергией спин-орбитального взаимодействия. Если при этом каналы прохождения тока преимущественно состоят из атомов доминирующей подрешетки $12 k$, следствием будет уменьшение результирующей намагниченности феррита. Найденные зависимости сдвига частот МСК от подводимой электрической мощности для многодоменного и насыщенного состояний образца позволили разделить вклады обоих эффектов и определить величину каждого из них. Предложенное феноменологическое описание исследованных эффектов, основанное на добавлении к свободной энергии ферромагнетика квадратичных по электрическому полю слагаемых, совместимых с кристалломагнитной симметрией материала, дало качественно согласующиеся с экспериментом результаты.

Данные эффекты могут найти применение при разработке управляемых током устройств аналоговой обработки сигналов миллиметрового диапазона, отличающихся уменьшенным временем перестройки и энергопотреблением. Особенно интересны результаты для 
многодоменного состояния, так как в этом случае в приборных конструкциях можно обойтись без применения громоздких магнитных систем.

\section{Список литературы}

[1] Berger L. // Phys. Rev. B. 1996. Vol. 54. N 13. P. 9353-9358. DOI: 10.1103/PhysRevB.54.9353

[2] Brataas A., Kent A.D., Ohno H. // Nat. Materials. 2012. Vol. 11. N 5. P. $372-381$. DOI: $10.1038 /$ nmat3311

[3] Ralph D.C., Stiles M.D. // JMMM. 2008. Vol. 320. N 7. P. 1190-1216. DOI: 10.1016/j.jmmm.2007.12.019

[4] Nan C.-W., Bichurin M.I., Dong S., Viehland D., Srinivasan G. // J. Appl. Phys. 2008. Vol. 103. N 3. P. 0311011-031101-35. DOI: 10.1063/1.2836410

[5] Eerenstein W., Mathur N., Scott J. // Nature. 2006. Vol. 442. P. 759-765. DOI: 10.1038 /nature05023

[6] Popkov A.F., Davydova M.D., Zvezdin K.A., Solov'yov S.V., Zvezdin A.K. // Phys. Rev. B. 2016. Vol. 93. N 9. P. 0944351-094435-5. DOI: 10.1103/PhysRevB.93.094435

[7] Fang C.M., Kools F., Metselaar R., DeWith G., de Groot R.A. // J. Phys.: Cond. Matter. 2003. Vol. 15. N 36. P. 6229-6237. DOI: 10.1088/0953-8984/15/36/311

[8] Izadkhah H., Zare S., Somu S., Vittoria C. // Appl. Phys. Lett. 2015. Vol. 106. N 14. P. 142905-1-142905-4.

DOI: $10.1063 / 1.4916102$

[9] Srinivasan G., Zavislyak I.V., Tatarenko A.S. // Appl. Phys. Lett. 2006. Vol. 89. N 15. P. 152508-1-152508-3. DOI: $10.1063 / 1.2360901$

[10] Song Y.-Y., Das J., Krivosik P., Mo N., Patton C.E. // Appl. Phys. Lett. 2009. Vol. 94. N 18. P. 182505-1-182505-3. DOI: $10.1063 / 1.3131042$

[11] Zavislyak I.V., Popov M.A., Srinivasan G. // Phys. Rev. B. 2016. Vol. 94. N 22. P. 224419-1-224419-10. DOI: 10.1103/PhysRevB.94.224419

[12] Schmid H. // J. Phys.: Cond. Matter. 2008. Vol. 20. N 43. P. 434201-1-434201-24. DOI: $10.1088 / 0953-8984 / 20 / 43 / 434201$

[13] Ахиезер А.И., Барьяхтар В.Г., Пелетминский С.В. Спиновые волны. М.: Наука, 1967. 36 с.

[14] Гуревич А.Г., Мелков Г.А. Магнитные колебания и волны. М.: Наука, 1994. 464 c.

[15] Aubert G. // J. Appl. Phys. 1982. Vol. 53. N 11. P. 8125-8129. DOI: $10.1063 / 1.330315$

[16] Newnham R.E. Properties of materials: anisotropy, symmetry, structure. Oxford: Oxford University Press, 2005. 391 p.

[17] Zaveta K. // Phys. Stat. Sol. 1963. Vol. 3. N 11. P. 2111-2118. DOI: $10.1002 / \mathrm{pssb} .19630031115$

[18] Данилов В.В., Зависляк И.В., Балинский М.Г. Спинволновая электродинамика. Киев: Лыбидь, 1991. 212 с.

[19] De Bitetto D.J. // J. Appl. Phys. 1964. Vol. 35. N 12. P. 3482 3487. DOI: $10.1063 / 1.1713254$

[20] Hellewege K.-H., Hellewege A.M. Landolt-Bornstein. New Series. N. Y:: Springer-Verlag, 1970. Vol. III/4b. Magnetic and other properties of oxides and related compounds. $666 \mathrm{p}$.

[21] Beleggia M., De Graef M., Millev Y.T. // J. Phys. D: Appl. Phys. 2006. Vol. 39. N 5. P. 891-899.

DOI: $10.1088 / 0022-3727 / 39 / 5 / 001$ 